\title{
Pneumonectomy for massive ventilated lung cysts
}

\author{
ED BATEMAN, DE WESTERMAN, RP HEWITSON, AD FERGUSON
}

From the Respiratory Clinic and the Department of Thoracic Surgery, Departments of Medicine and Surgery, University of Cape Town and Groote Schuur Hospital, Cape, South Africa.

Surgical treatment of bullous lung disease is reserved for a small minority of patients with severe breathlessness in whom it can be shown that the localised bullous disease is contributing to the symptoms. ${ }^{1}$ The disability usually arises from the compression of adjacent normal or near normal lung tissue and this may be relieved by an operation. ${ }^{2}$ Occasionally an operation is advised to remove well-ventilated but non-perfused cysts which cause excessive dead space ventilation and aggravate dyspnoea by increasing the work of breathing. This type of defect is rarely found in its pure form. Surgical procedures are usually limited to local excision or plication of bullae or small wedge resections. Lobectomy is sometimes necessary, but pneumonectomy has rarely been resorted to for removal of cysts.

We report here a patient with cystic lung disease in whom disproportionate dead space ventilation contributed significantly to his dyspnoea and who benefited considerably from pneumonectomy.

\section{Case report}

A 28-year-old labourer presented with a history of gradually increasing breathlessness since being treated for pulmonary tuberculosis six years earlier. $\mathrm{He}$ had not worked for a year, and for the previous six months had been dyspnoeic on minimal exertion. He had required admission to hospital on two occasions during this period for severe dyspnoea. He had not smoked for six years. On examination he was cyanosed and tachypnoeic at rest (32 breaths/minute) and became distressed while dressing himself and walking a few metres. Chest movements were symmetrical though reduced. The left chest was hyperresonant with loud, harsh, amphoric breath sounds throughout. Over the right side there were patchy crackles and variable inspiratory and expiratory wheezes. There was no finger clubbing or evidence of cor pulmonale. The chest radiograph (fig 1) confirmed the presence of large bullae in the left hemithorax. There was a hypertransradiant area in the right upper zone and the remainder of the lung showed abnormal streaking, suggesting fibrosis. The electrocardiograph was normal apart from right axis deviation (QRS axis $\left.+100^{\circ}\right)$.

A ventilation lung scan (Xenon 133) (fig 2) showed equal ventilation of both lungs including the large cysts on the left side. Neither wash-in nor wash-out was delayed on either side. The perfusion scan (Technetium

Address for reprint requests: Dr AD Ferguson, Respiratory Clinic, Groote Schuur Hospital, Observatory 7925, Cape, South Africa.
99 macro-aggregated albumin) (fig. 2) demonstrated that only $7 \%$ of the pulmonary blood flow was distributed to the left lung. In both scans there was impaired uptake in the right apical region. Lung volume measurements and ventilatory tests indicated the presence of moderate obstructive and restrictive lung disease. The total lung capacity was reduced and the residual volume to total lung capacity ratio (RV/TLC\%) was increased. The functional residual capacity (FRC) was not substantially different when measured by the multi-breath helium dilution method or by the whole-body plethysmograph. A progressive exercise test on a treadmill with measurement of expired gases was performed. Arterial gas tensions were sampled at rest and during the first minute at the maximum level of exercise. The patient could tolerate 2.5 kilometres per hour on the level but could only maintain this for two minutes, thus precluding a steady state measurement. This corresponded with an

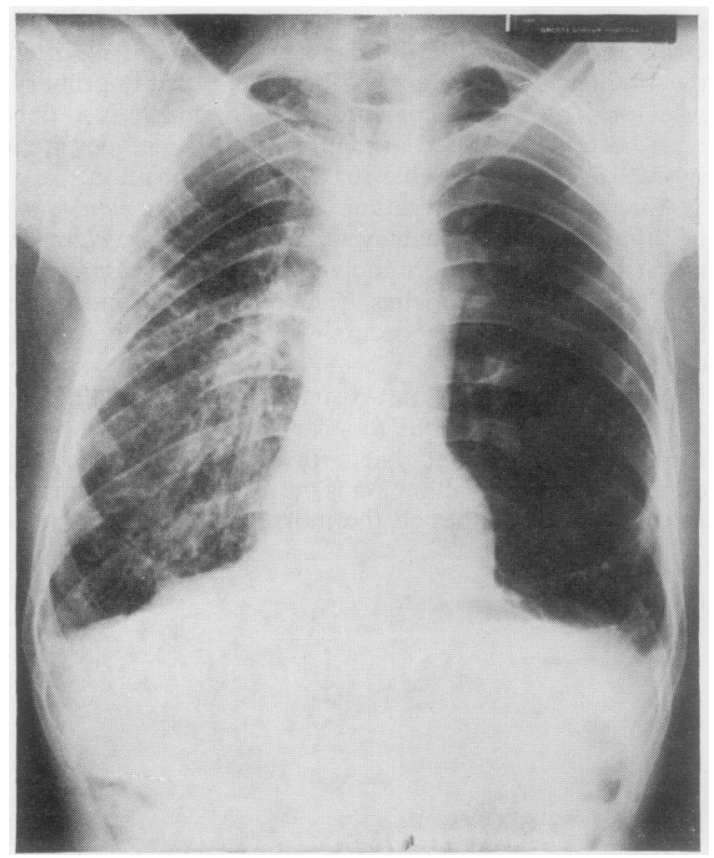

Fig 1 Chest radiograph showing multiple large cysts in left lung field, with a smaller transradiant area in right upper zone. Right lung streaking suggests fibrosis. 


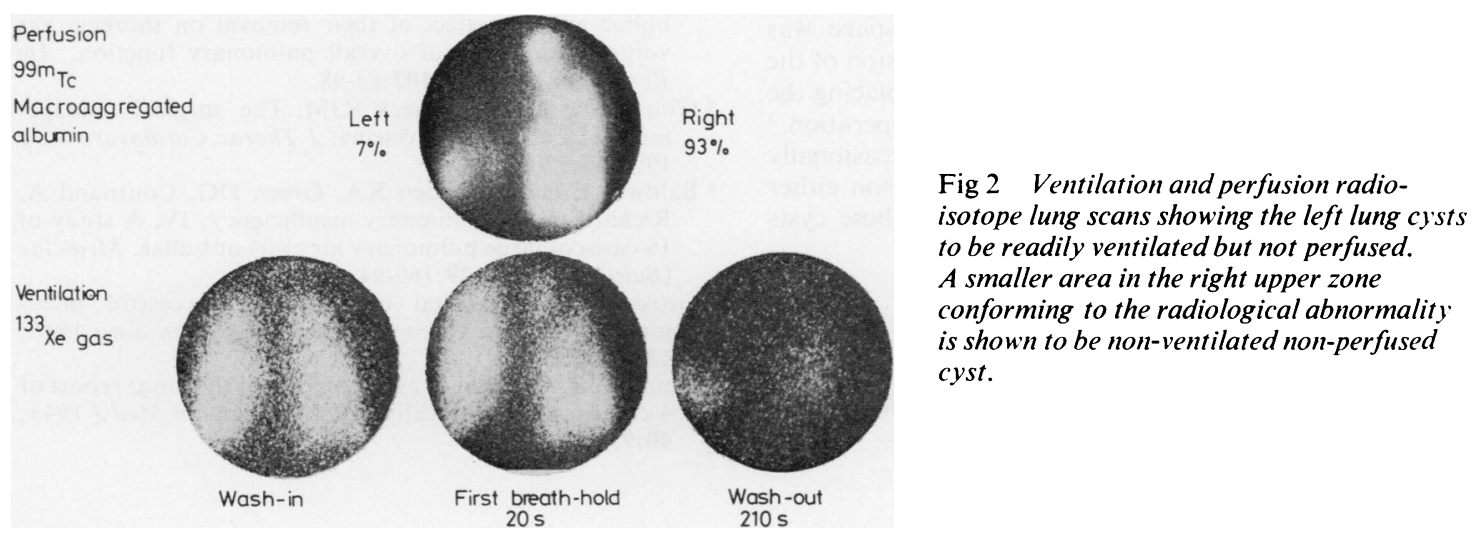

oxygen uptake (ن்2) of 0.89 litres/minute and was achieved with a disproportionate increase in minute ventilation ( $\dot{\mathrm{VE}}$ ) to 44 litres at a respiratory frequency (rr) of 50. Arterial blood gas analyses showed an abnormal alveolar-arterial gradient (A-a) at rest which widened during exercise. Hypercarbia and acidosis also developed. The markedly increased dead-space to tidal volume ratio $(\mathrm{Vd} / \mathrm{Vt})$ was unchanged.

As clinical examination, lung scan, and pulmonary function tests all confirmed the presence of a large ventilated dead-space, it was argued that obliteration of the space would result in improvement of the patient's effort tolerance by reducing the work of breathing. Thoracotomy was therefore performed with the intention of plicating the bullae or closing the communications between these and the bronchial tree. At operation two very large cysts were found replacing most of the left lung. These were opened and multiple communications with the major bronchi were seen and closed. Despite this, a large leak persisted. For this reason and also since the remaining lung tissue was fibrous and unable to expand, left pneumonectomy was performed. Histology of the removed lung showed the cysts to be partially lined with respiratory epithelium. There was marked fibrosis throughout the lung but there were no features of active tuberculosis. After the operation, the patient did not need assisted ventilation and made an uneventful recovery.

Lung volumes and ventilation were measured one month after operation. These confirmed that a readily ventilated portion of lung had been excised. There was a fall not only in the RV and TLC but also in the VC and $\mathrm{FEV}_{1}$. The patient achieved a similar maximal $\dot{\mathrm{O}} 2$ during the exercise study, and the end point of the test was at a similar respiratory rate and level of arterial oxygen tension as previously. The corresponding grade of exercise at this $\mathrm{VO} 2$ had, however, improved to 3.5 kilometres per hour on a $5 \%$ incline. There was a $40 \%$ reduction in $\dot{V} E$ and an equivalent reduction in $\mathrm{Vt}$. Hypercarbia and acidosis did not develop and the $\mathrm{Vd} / \mathrm{Vt}$ ratio was significantly reduced. Subjectively the patient was no longer breathless at rest and could manage one flight of stairs with ease. Eighteen months later the clinical improvement has been maintained and he is working as a fulltime gardener.

\section{Discussion}

The object of preoperative assessment of bullous lung disease is to demonstrate either that normal lung is being compressed or that there is an increase in localised dead-space ventilation. Lung scanning, lobar gas sampling, bronchospirometry, and exercise studies have added to the accuracy of assessment of the degree of ventilation of bullae. In contrast, when the bullae are shown to be non-ventilated, these tests give little indication of the amount of compressed potential functioning lung. Most information can be obtained from the chest radiograph ${ }^{3}$ and the usual lung function tests. The $\mathrm{CO}$ transfer factor in particular gives an indication of the degree of generalised emphysema. ${ }^{2}$ Ventilated and non-ventilated cysts can coexist in the same patient and the combination of these tests provides a means of separating the two pathophysiological effects.

Our case is a rare example in which the dominant reversible disability was dead-space ventilation. The particular features in keeping with the large dead-space were the symmetrical chest movement coupled with the loud amphoric breath sounds on clinical examination, hypercarbia, despite an $\mathrm{FEV}_{1}$ of 1.34 litres and a close correlation between the FRC measured by the body plethysmograph and the helium dilution method. Other typical features were the obvious ventilation-perfusion mismatching shown on the lung scans, the excessive $\dot{V} E$ necessary for a modest oxygen uptake, the worsening hypercarbia on exercise, and the large dead-space. The dramatic fall after operation in $\dot{V} E$ and $V t$ when these are related to $\dot{\mathrm{VO} 2}$ is evidence that the benefit to the patient was achieved through reduction in the work of breathing. This improvement occurred despite the reduction in $\mathrm{FEV}_{1}$, whereas clinical improvement where compression of the lung is reversed is associated

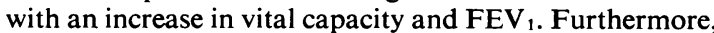
the Kco (carbon dioxide transfer per litre of lung volume) rose after operation confirming that ventilated lung not taking part in gas exchange had been removed.

Pneumonectomy has rarely been used in the management of bullous emphysema and cystic lung disease as an 
indication seldom occurs. Baldwin et $a l^{4}$ reported a case similar to ours in which the increased dead-space was demonstrated by bronchospirometry. Compression of the opposite lung by giant non-ventilated cysts displacing the mediastinum is another indication for operation. ${ }^{4}$ Congenital cystic disease of the lung occasionally necessitates pneumonectomy for the same reason either in children ${ }^{5}$ or in adults. ${ }^{6}$ More commonly these cysts are removed because of complicating infection.

\section{References}

${ }^{1}$ Hugh-Jones $\mathrm{P}$, Whimster $\mathrm{W}$. The aetiology and management of disabling emphysema. Am Rev Respir Dis 1978;117:343-7.
2 Pride NB, Barter CE, Hugh-Jones P. The ventilation of bullae and the effect of their removal on thoracic gas volumes and tests of overall pulmonary function. $\mathrm{Am}$ Rev Respir Dis 1973;107:83-98.

${ }^{3}$ Gunstensen J, McCormack RJM. The surgical management of bullous emphysema. $J$ Thorac Cardiovasc Surg $1973 ; 65: 920-5$.

${ }^{4}$ Baldwin E de F, Harden KA, Green DG, Cournand A Richards DW. Pulmonary insufficiency: IV. A study of 16 cases of large pulmonary air cysts or bullae. Medicine (Baltimore) 1950;29:169-94.

5 Gross RE. Congenital cystic lung. Successful pneumonectomy in a three-week-old baby. Ann Surg 1946; 123:229-37.

6 Rumel WR. Congenital cystic disease of the lung: report of 4 cases treated surgically. Rocky Mountain Med J 1943; 40:95-103. 